ARTICLE

https://doi.org/10.1038/s41467-019-12596-6

\title{
Rational design of crystalline two-dimensional frameworks with highly complicated topological structures
}

Rong-Ran Liang ${ }^{1,3}$, Shun-Qi Xu ${ }^{1,3}$, Lei Zhang ${ }^{2,3}$, Ru-Han A ${ }^{1}$, Pohua Chen ${ }^{2}$, Fu-Zhi Cui ${ }^{1}$, Qiao-Yan Qi ${ }^{1}$, Junliang Sun (1) ${ }^{2 \star} \&$ Xin Zhao ${ }^{1 *}$

Constructing two-dimensional (2D) polymers with complex tessellation patterns via synthetic chemistry makes a significant contribution not only to the understanding of the emergence of complex hierarchical systems in living organisms, but also to the fabrication of advanced hierarchical materials. However, to achieve such tasks is a great challenge. In this communication we report a facile and general approach to tessellate 2D covalent organic frameworks (COFs) by three or four geometric shapes/sizes, which affords 2D COFs bearing three or four different kinds of pores and increases structural complexity in tessellations of $2 \mathrm{D}$ polymers to a much higher level. The complex tessellation patterns of the COFs are elucidated by powder X-ray diffraction studies, theoretical simulations and high-resolution TEM.

\footnotetext{
${ }^{1}$ Key Laboratory of Synthetic and Self-Assembly Chemistry for Organic Functional Molecules, Center for Excellence in Molecular Synthesis, Shanghai Institute of Organic Chemistry, University of Chinese Academy of Sciences, Chinese Academy of Sciences, 345 Lingling Road, Shanghai 200032 , China. ${ }^{2}$ Beijing National Laboratory for Molecular Science (BNLMS), State Key Laboratory of Rare Earth Materials Chemistry and Applications, College of Chemistry and Molecular Engineering, Peking University, Beijing 100871, China. ${ }^{3}$ These authors contributed equally: Rong-Ran Liang, Shun-Qi Xu, Lei Zhang. *email: junliang.sun@pku.edu.cn; xzhao@sioc.ac.cn
} 
T he existence of complex hierarchical structures widely found in biological materials is not only one of the most miraculous phenomena in the nature, but also a key foundation for biomaterials to achieve functions ${ }^{1,2}$. With an aim to mimic natural materials in functions as well as in structures, fabrication of materials with hierarchical structures has drawn considerable attention and great progress has been achieved over the past decades ${ }^{3-5}$. However, so far the researches have mainly focused on zero-dimensional (0D), one-dimensional (1D), and three-dimensional (3D) hierarchical materials ${ }^{6-10}$. In terms of two-dimensional (2D) hierarchical structures, they are closely related to an intriguing subject in mathematics, namely tessellation, which refers to completely tiling a plane by one or more geometric shapes without overlaps and gaps ${ }^{11}$. Tessellation patterns are not only widely observed in the nature, but also frequently used in art, architecture and manufacturing. However, the development of tessellations in 2D materials is just in the bud. As the archetype of $2 \mathrm{D}$ materials, graphene exhibits a simple hexagonal tilling ${ }^{12,13}$. Similarly, simple tessellation patterns are also observed for the other inorganic 2D materials such as $\mathrm{MoS}_{2}$, h-BN and phosphorene ${ }^{14,15}$, due to the limitation of their structural motifs. On the other hand, 2D polymers, which are composed entirely of organic building blocks, provide tremendous structural variabilities. In this context, $2 \mathrm{D}$ covalent organic frameworks (COFs), an emerging class of crystalline porous materials precisely integrated with periodical organic units through covalent bonds in a plane and interlayer stacking to form layered 3D crystals via non-covalent interactions ${ }^{16,17}$, have been widely recognized as general $2 \mathrm{D}$ polymers, while a stricter definition of $2 \mathrm{D}$ polymers refers to those isolated as monolayers ${ }^{18,19}$.

Thanks to the advantages of their conjugated skeletons and porosities, $2 \mathrm{D}$ COFs have been extensively exploited to be functional materials with versatile applications ranging from gas separation and storage ${ }^{20-25}$, catalysis $^{26-30}$, drug delivery ${ }^{31,32}$, sensing $^{33-35}$, proton conduction ${ }^{36,37}$, to optoelectronic devices ${ }^{38-44}$. However, despite the progress achieved, the topological structures of 2D COFs are still limited to simple tessellation patterns, which are mainly tiled by only one geometric shape (usually hexagon or tetragon), leading to COFs with homogeneous porosities ${ }^{45-47}$. In 2014, we reported a Kagome-type 2D COF in which hexagonal mesopores and triangular micropores alternately and periodically distribute $^{48}$. Following this dual-pore COF, triple-pore COFs were successful achieved in 2016, which increased the complexity in the tessellations of $2 \mathrm{D}$ COFs to a higher level ${ }^{49}$. Such heteropore COFs possess hierarchical structures and exhibit heterogeneous porosities, which benefit for exploiting new properties, functions and applications $s^{50-57}$. For example, making use of the advantage that the different kinds of pores can be independently modified and functionalized, distinct functions can be introduced into different types of pores and thus advanced materials with integrated functions can be fabricated. Moreover, different guest molecules, even those incompatible with each other, might be encapsulated in the different types of pores of one COF due to their different pore environments. On the other hand, hierarchically porous materials have already been found to exhibit some advantages over homogeneous ones, such as minimizing diffusion barriers, improving mass transport, and increasing distribution of active sites ${ }^{3,4}$. Indeed, the inimitable advantages of COFs with hierarchical porosity were demonstrated in recent research led by $\mathrm{Ma}$, which clearly showed that a Kagome-shaped dual-pore COF outperformed the COF with uniform porosity in enzyme catalytic performance by loading the enzyme in the large hexagonal pores while making the small triangular pores free to transport reactants and products ${ }^{58}$. Despite the great potential applications, COFs with complex tessellation patterns have remained very difficult to achieve.
We herein report a facile and general approach to fabricate $2 \mathrm{D}$ COFs which bear three or four different kinds of pores in one framework. To the best of our knowledge, the latter is the highest level of hierarchy and complexity held by a 2D COF reported so far, demonstrating a successful step further to challenge the structural complexity in the tessellations of $2 \mathrm{D}$ polymers.

\section{Results}

Design and synthesis of the COFs. The approach has been developed by taking advantage of multiple-linking-site $e^{59}$ and desymmetrization ${ }^{60}$ strategies simultaneously. As illustrated in Fig. 1, desymmetrization of a $\mathrm{C}_{3}$-symmetric double-linking-site building block leads to a monomer with $\mathrm{C}_{2}$-symmetry. The condensation of it with linear monomers is expected to afford COFs bearing three different kinds of pores (Fig. 1, lower left). Further desymmetrizing this monomer produces an inequilateral building block, which could be used to tessellate $2 \mathrm{D}$ frameworks with four different kinds of pores through assembling it with linear linkers (Fig. 1, right).

Directed by this strategy, monomers TPM and FPM were designed and synthesized (Supplementary Fig. 1). 1,4-diaminobenzene $(\mathrm{DAB})$ and benzidine (BZ) were chosen as the linear monomers. On the basis of the above design, triple-pore 2D COFs (Tri-COF-DAB and Tri-COF-BZ) and tetrad-pore 2D COFs (Tetra-COF-DAB and Tetra-COF-BZ) will be produced by the condensations of TPM or FPM with the diamines, respectively (see Supplementary Fig. 2 for their structures). Tri-COF-DAB was synthesized as yellow crystallites by heating TPM and DAB in a mixture of 1,4-dioxane, mesitylene and acetic acid (aq., $6 \mathrm{M})(5 /$ $5 / 1, v / v / v)$ in a sealed glass ampoule at $120{ }^{\circ} \mathrm{C}$ for 3 days. Under the similar solvothermal condition, Tetra-COF-DAB was prepared from the condensation of FPM and DAB.

Spectroscopy and thermal stability analysis of the COFs. Fourier transform infrared spectroscopy (FTIR) confirmed formation of imine bonds in the polymers, as evidenced by the appearance of vibration bands of $\mathrm{C}=\mathrm{N}$ units in their spectra (Supplementary Figs. 3, 4). High degrees of polymerizations of the condensation reactions were indicated by the dramatic attenuation of the vibration bands of $\mathrm{NH}_{2}$ groups of DAB. Compared with TPM and FPM, the intensities of $\mathrm{C}=\mathrm{O}$ stretching vibration peaks of the COFs attenuated dramatically, further confirming the almost complete conversion of the monomers. ${ }^{13} \mathrm{C}$ crosspolarization magic angle spinning solid-state NMR spectroscopy was performed for the COFs to characterize the connections of their frameworks. The formation of imines was indicated by the resonance signals of $\mathrm{C}=\mathrm{N}$ bonds, which were observed at 157.3 and $155.8 \mathrm{ppm}$ in the spectra of Tri-COF-DAB and Tetra-COFDAB, respectively (Supplementary Figs. 5, 6). Thermogravimetric analysis (TGA) profiles show less than $4.3 \%$ weight loss when the temperature increased to $400^{\circ} \mathrm{C}$, indicating good thermal stabilities of these polymers (Supplementary Fig. 7).

PXRD analysis and structural modeling of the COFs. To elucidate the structure of Tri-COF-DAB, the model of the triple-pore $\mathrm{COF}$ with monolayer was firstly predicted on the basis of reticular chemistry by Accelarys Materials Studio 7.0 software. Then two possible stacking models of the COF layers were established and optimized (Supplementary Fig. 8): (i) eclipsed stacking (AA, space group $P 2)$, (ii) staggered stacking ( $\mathrm{AB}$, space group $P 1$ ), based on which the simulated powder X-ray diffraction (PXRD) patterns were obtained (Fig. 2b, c). As for the experimental PXRD profile of Tri-COF-DAB, an intense peak is observed at $2 \theta=$ $2.69^{\circ}$, accompanied by a series of relatively weak peaks appearing at $4.73^{\circ}, 5.47^{\circ}, 7.23^{\circ}$, and $8.29^{\circ}$ (Fig. $2 \mathrm{a}$ ). It should be pointed out 


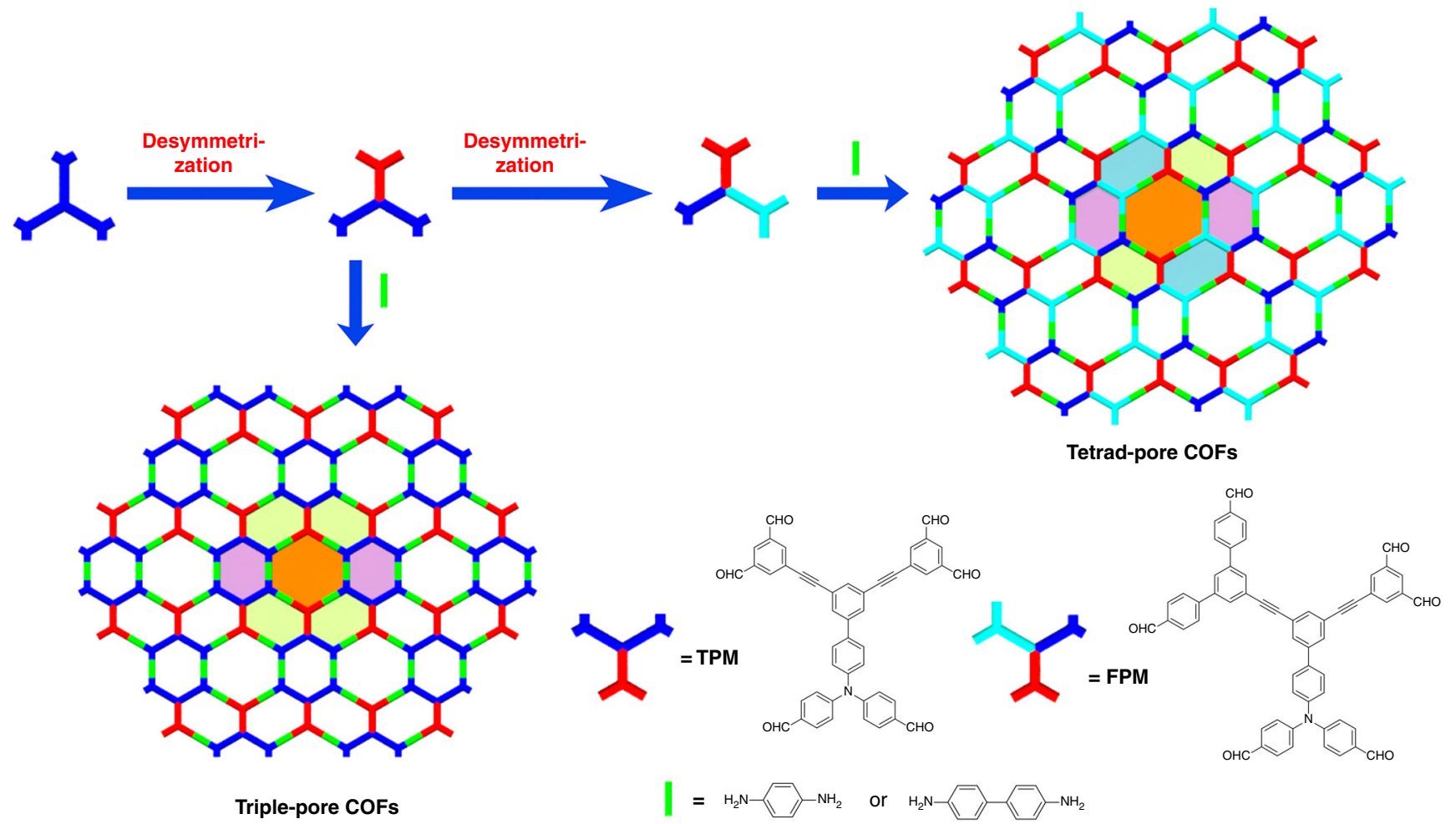

Fig. 1 Design strategy. Cartoon representation of the fabrication of triple-pore and tetrad-pore COFs
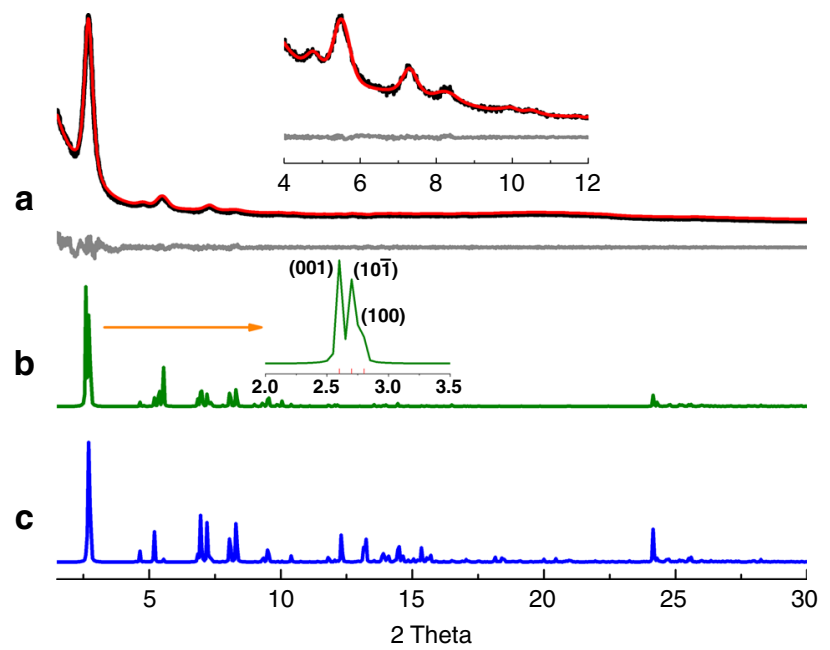

Fig. 2 PXRD profiles of Tri-COF-DAB. a Experimental (black) and Pawley refined (red) PXRD patterns and difference plot (gray) between them, b simulated PXRD patterns with $A A$ stacking, and c $A B$ stacking

that, in its simulated PXRD pattern with AA stacking, three peaks corresponding to $\left(\begin{array}{lll}0 & 0 & 1\end{array}\right),\left(\begin{array}{lll}1 & 0 & \overline{1}\end{array}\right)$ and $\left(\begin{array}{lll}1 & 0 & 0\end{array}\right)$ reflections are very close to each other (Fig. 2b inset and Supplementary Fig. 9a). Due to the broadening and overlapping of these peaks, the three peaks could not be distinguished each other in the experimental PXRD pattern and thus just appear as a single peak centered at $2.69^{\circ}$. A close comparison indicates that the experimental PXRD pattern is in good agreement with that of the simulated triple-pore COF with AA stacking model. Furthermore, the lower total energy of AA stacking $(242.72 \mathrm{kcal} / \mathrm{mol})$ than that of AB stacking $(515.14$ $\mathrm{kcal} / \mathrm{mol}$ ) also indicates that Tri-COF-DAB adopts AA stacking.

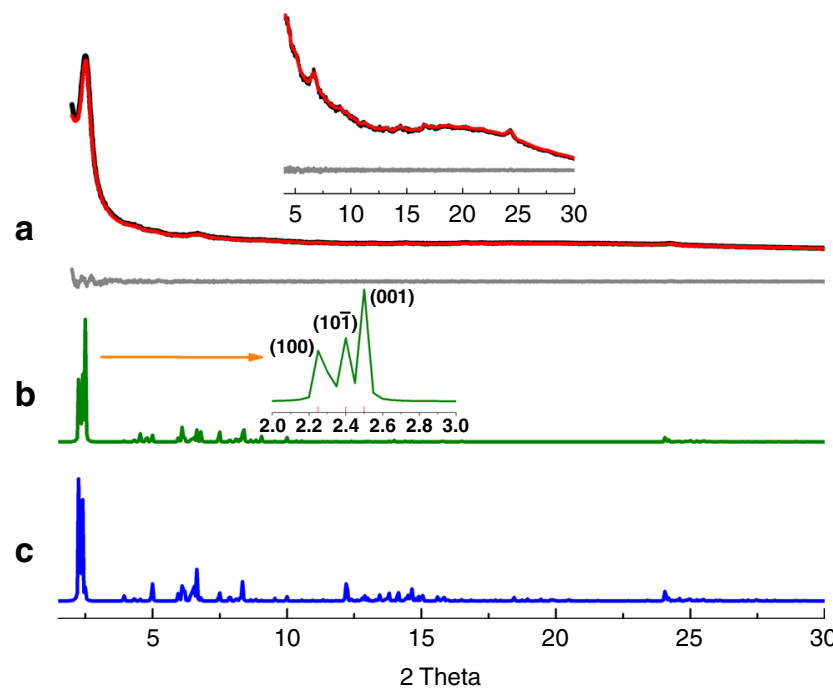

Fig. 3 PXRD profiles of Tetra-COF-DAB. a Experimental (black) and Pawley refined (red) PXRD patterns and difference plot (gray) between them, b simulated PXRD patterns with $A A$ stacking, and $\mathbf{c} A B$ stacking

Pawley refinement was performed to yield unit cell parameters of $a=36.79 \AA, b=3.68 \AA, c=39.23 \AA, \alpha=\gamma=90^{\circ}, \beta=120^{\circ}$, with $R_{\mathrm{wp}}=3.16 \%$ and $R_{\mathrm{p}}=2.36 \%$. The coincidence of the refined PXRD pattern and experimental data could be evaluated by the difference plot. As revealed by Fig. 2a, the refined PXRD pattern matches with the experimentally obtained PXRD pattern quite well. Based on the PXRD results, the triple-pore structure of TriCOF-DAB is confirmed.

The crystal structure of Tetra-COF-DAB was elucidated by the same process as above. It exhibits a strong peak at $2.51^{\circ}$, along with several weak peaks at $5.19^{\circ}, 6.66^{\circ}, 9.00^{\circ}$, and $24.25^{\circ}$ (Fig. 3a). Its experimental PXRD pattern matches well with the simulated 

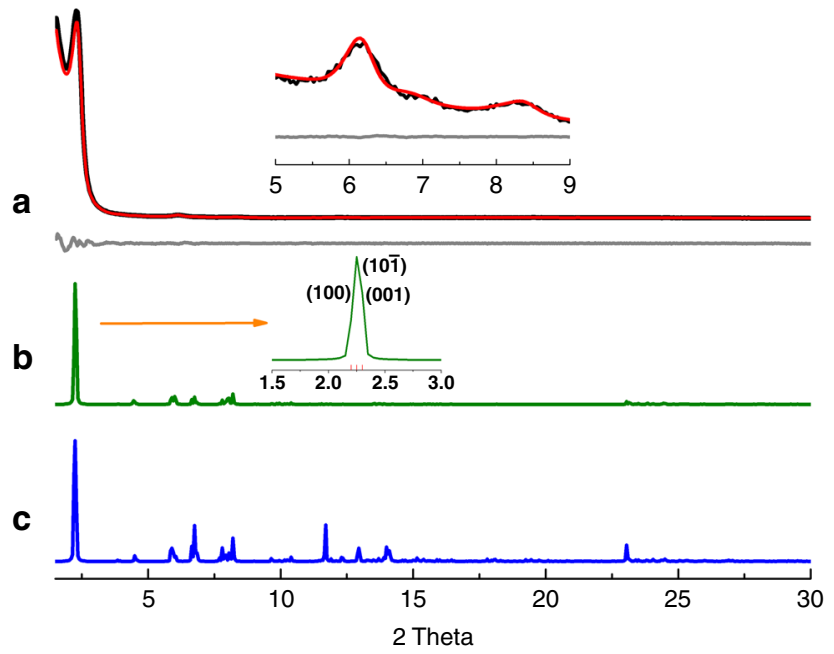

Fig. 4 PXRD profiles of Tri-COF-BZ. a Experimental (black) and Pawley refined (red) PXRD patterns and difference plot (gray) between them, b simulated PXRD patterns with $A A$ stacking, and $\mathbf{c} A B$ stacking
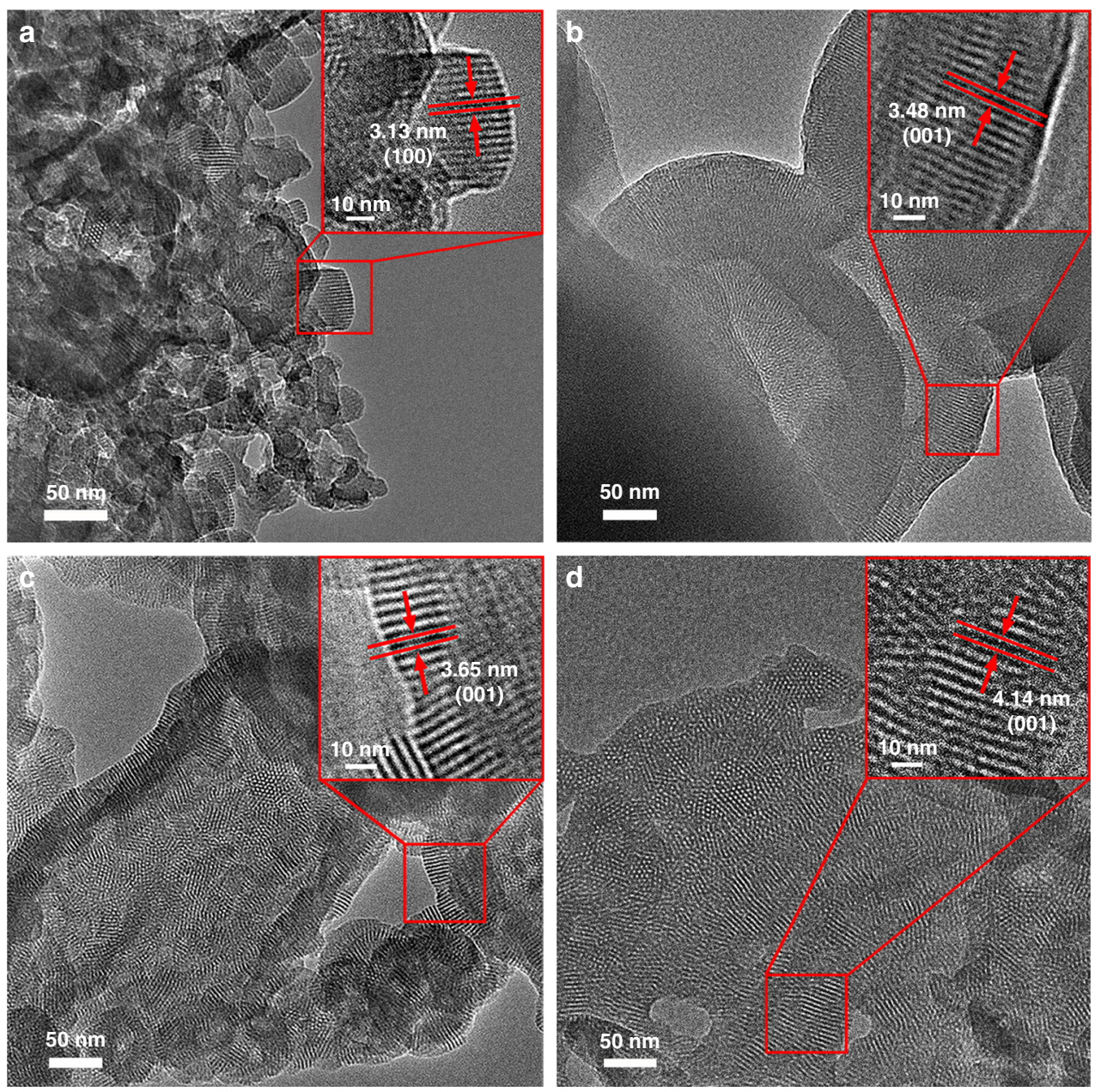

Fig. 6 TEM analysis. TEM images of a Tri-COF-DAB, b Tetra-COF-DAB, $\mathbf{c}$ Tri-COF-BZ, and $\mathbf{d}$ Tetra-COF-BZ. The insets in the upper right corners are magnified images from their corresponding red squares

PXRD pattern of the predicted tetrad-pore COF with AA stacking (Fig. 3b), confirming that Tetra-COF-DAB holds a structure bearing four different kinds of pores. The lower total energy of AA stacking $(265.64 \mathrm{kcal} / \mathrm{mol})$ than that of $\mathrm{AB}$ stacking $(623.67$ a

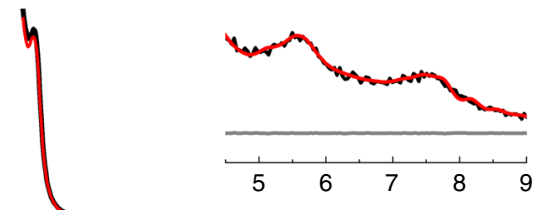

b

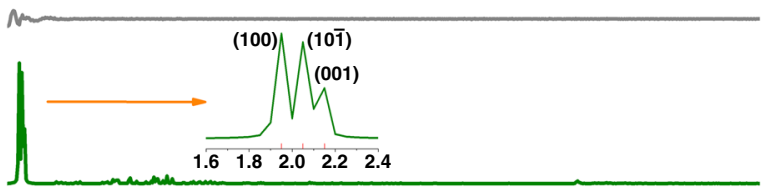

C

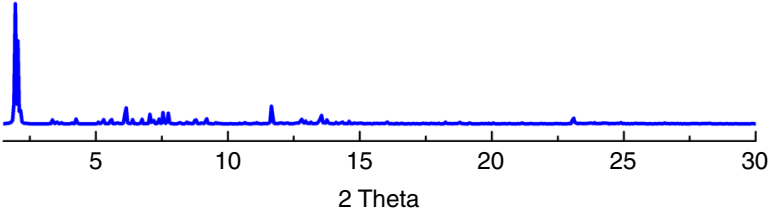

Fig. 5 PXRD profiles of Tetra-COF-BZ. a Experimental (black) and Pawley refined (red) PXRD patterns and difference plot (gray) between them, b simulated PXRD patterns with AA stacking, and c AB stacking $\mathrm{kcal} / \mathrm{mol}$ ) further supports that Tetra-COF-DAB adopts AA stacking. Pawley refinement gave lattice parameters of $a=44.94$ $\AA ̊ ., b=3.69 \AA, c=40.89 \AA, \alpha=\gamma=90^{\circ}, \beta=120^{\circ}$, with $R_{\mathrm{wp}}=$ $1.59 \%$ and $R_{\mathrm{p}}=1.03 \%$. The difference plot indicates that PXRD 
pattern obtained through Pawley refinement well reproduces the experimental pattern (Fig. 3a).

To illustrate the generality of this approach, another two triplepore and tetrad-pore COFs with larger pores, namely Tri-COFBZ and Tetra-COF-BZ, were also fabricated from the condensations of benzidine with TPM and FPM, respectively (Supplementary Fig. 2). Obtention of the expected COFs bearing three or four different kinds of pores has also been confirmed by comparing the experimental PXRD patterns with the simulated patterns of the predicted models of triple-pore and tetrad-pore COFs (Figs. 4-5). The lattice parameters of Tri-COF-BZ were yielded by Pawley refinement to be $a=45.95 \AA, b=3.85 \AA, c=$ $44.59 \AA, \alpha=\gamma=90^{\circ}, \beta=120^{\circ}$, with $R_{\mathrm{wp}}=1.57 \%$, and $R_{\mathrm{p}}=$ $1.08 \%$. For Tetra-COF-BZ, the unit cell parameters of $a=52.50$ $\AA, b=3.85 \AA, c=47.86 \AA, \alpha=\gamma=90^{\circ}, \beta=120^{\circ}$, with $R_{\mathrm{wp}}=$ $1.50 \%$ and $R_{\mathrm{p}}=1.08 \%$ were achieved. The compositions of the two COFs were intensively characterized with FTIR and solidstate $\mathrm{CP} / \mathrm{MAS}{ }^{13} \mathrm{C}$ NMR spectroscopies, the results of which well support the formation of imine-linked polymers with high degrees of polymerizations (Supplementary Figs. 10-13).

SEM and TEM analysis of the COFs. Scanning electron microscopy (SEM) and transmission electron microscopy (TEM) were further conducted to investigate the morphologies and interior structures of the as-obtained COFs. SEM images showed spherical morphologies for all the four COFs (Supplementary Fig. 14). TEM images also indicated the formation of spherical aggregates (Supplementary Fig. 15), which match well with the results of the SEM study. A close analysis of the TEM images revealed that each sphere is polycrystalline, which consists of many 2D flakes. All of these flakes are resolved to be single crystals in good crystallinity by clear lattice fringes (Fig. 6), further indicating high crystallinities of these COFs.

To verify the reliability of the structural identifications of the COFs, which are based on the PXRD results above, detailed TEM study was carried out. Firstly, the fine structures of the lattice fringes in the TEM images were further examined. As shown in Fig. 6, the distances between the lattice fringes were measured to be $3.13,3.48,3.65$, and $4.14 \mathrm{~nm}$ for Tri-COF-DAB, Tetra-COF$\mathrm{DAB}$, Tri-COF-BZ, and Tetra-COF-BZ, respectively, which are in good agreement with the $d$-spacing of $\left(\begin{array}{lll}0 & 0 & 1\end{array}\right)$ or $\left(\begin{array}{lll}1 & 0 & 0\end{array}\right)$ reflection of the predicted triple-pore and tetrad-pore COF models with AA stacking $(3.19,3.54,3.86$, and $4.14 \mathrm{~nm}$ for Tri-COF-DAB, TetraCOF-DAB, Tri-COF-BZ, and Tetra-COF-BZ, respectively), indicating the validity of the predicted COF structures.

Furthermore, as shown in Figs. $7 \mathrm{a}, \mathrm{b}$ and $8 \mathrm{a}, \mathrm{b}$, network structures could be observed in the TEM images of these four COFs. To obtain clearer visualizations of the networks, highresolution (HR) TEM images of the regions marked by the red squares in Figs. $7 \mathrm{a}, \mathrm{b}$ and Fig. 8a, b were further recorded at a higher resolution to produce Figs. $7 c, d$ and $8 c$, d, respectively. As shown in these figures, clear reticular structures with approximately hexagonal pores could be observed. The distances between the centers of the two adjacent pores are $3.5 \pm 0.1 \mathrm{~nm}, 4.1 \pm 0.1$ $\mathrm{nm}, 4.2 \pm 0.1 \mathrm{~nm}$, and $4.8 \pm 0.2 \mathrm{~nm}$ for Tri-COF-DAB, TetraCOF-DAB, Tri-COF-BZ, and Tetra-COF-BZ, respectively, which are in good agreement with the unit cell of $a$ or $c$ derived from the Pawley refinements of the PXRD data, indicating that the
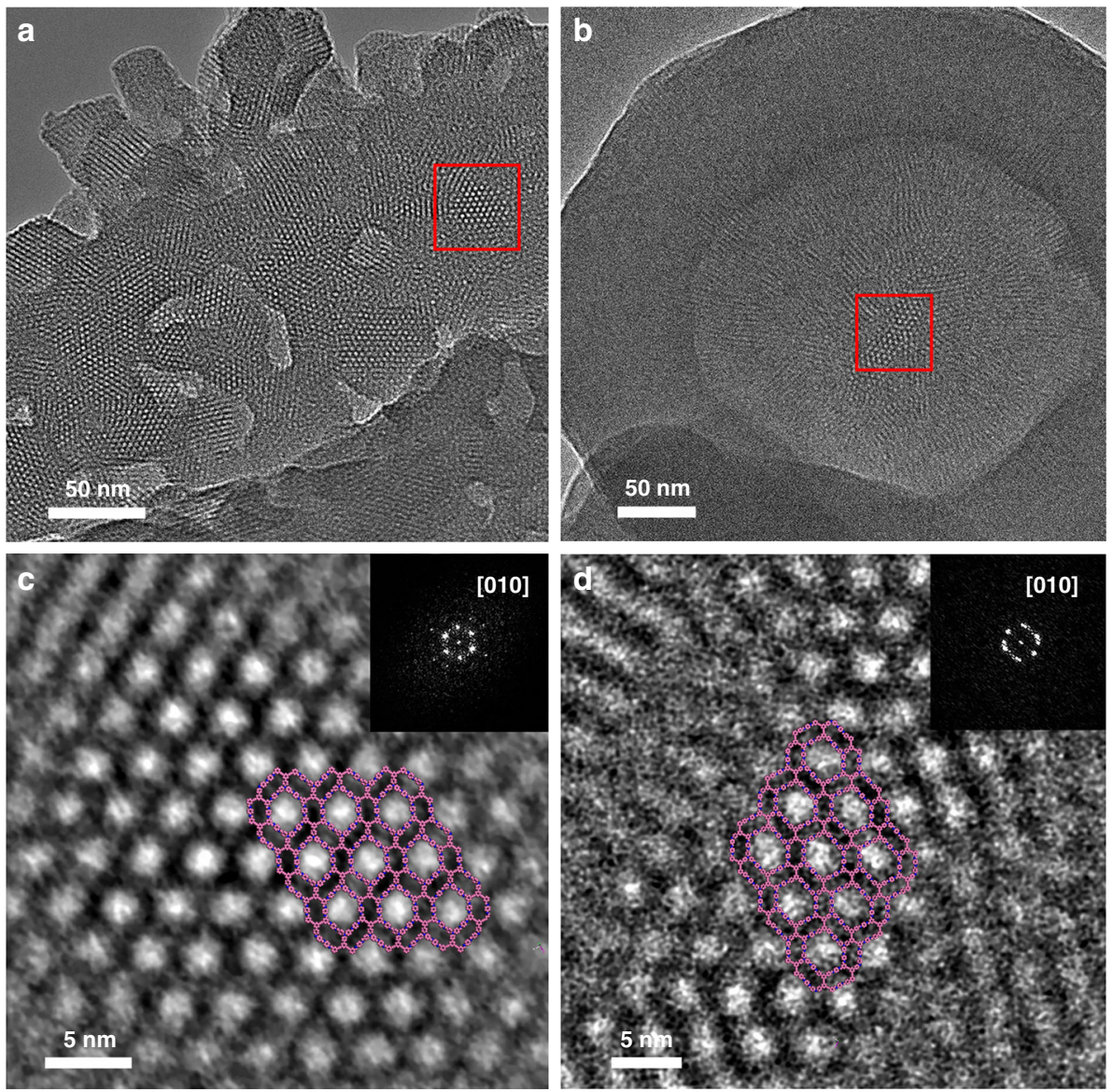

Fig. 7 TEM analysis. TEM images of $\mathbf{a}$ Tri-COF-DAB and $\mathbf{b}$ Tetra-COF-DAB, and HR-TEM images of $\mathbf{c}$ Tri-COF-DAB and $\mathbf{d}$ Tetra-COF-DAB focusing on the regions marked by the red squares in $\mathbf{a}$ and $\mathbf{b}$ at a higher resolution. The images inserted in the upper right corners are their corresponding FFT patterns, and the structure projections in HR-TEM images are along the $b$-axis and the structural models are inserted in the middle 

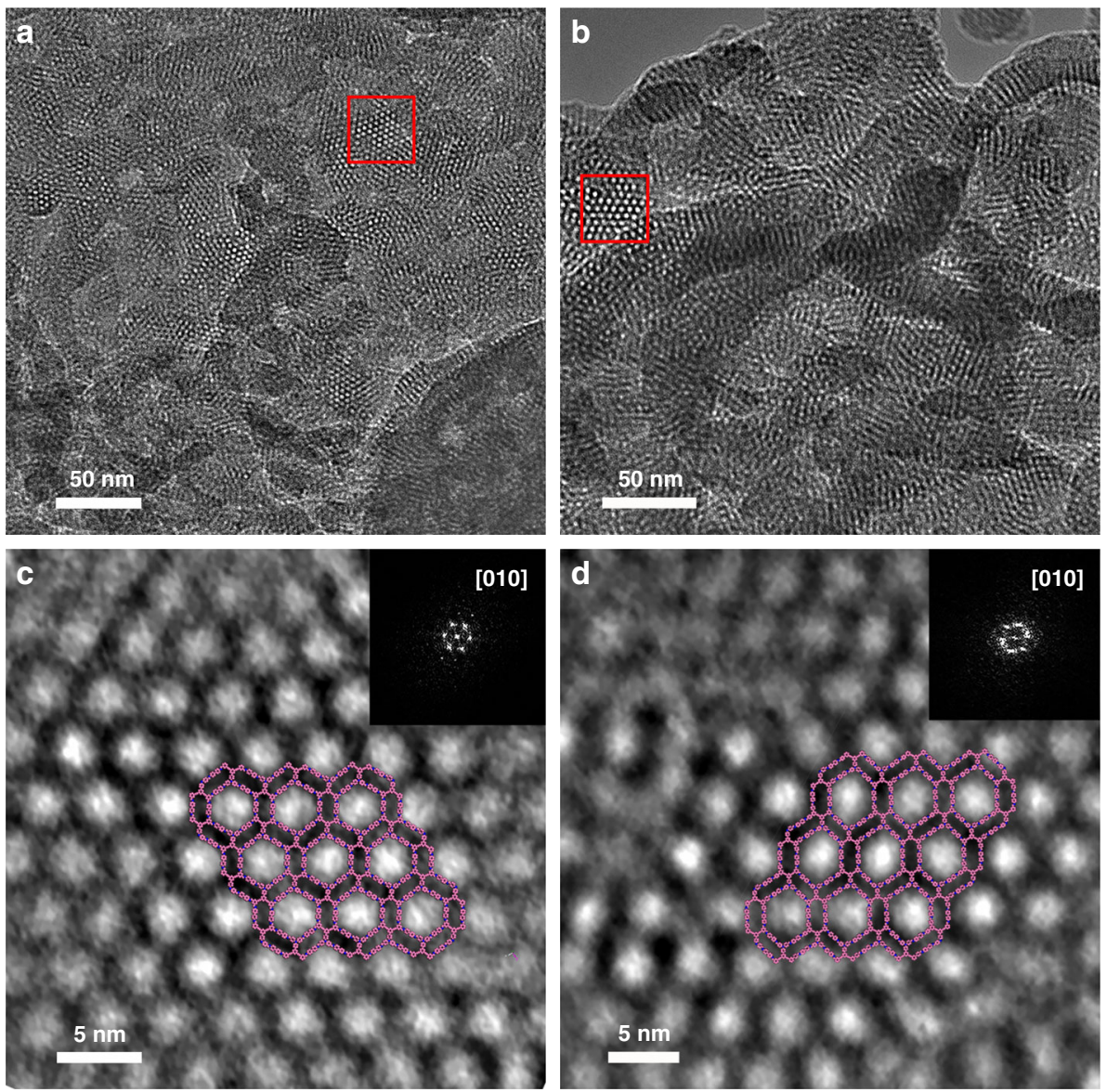

Fig. 8 TEM analysis. TEM images of a Tri-COF-BZ and $\mathbf{b}$ Tetra-COF-BZ, and HR-TEM images of $\mathbf{c}$ Tri-COF-BZ and $\mathbf{d}$ Tetra-COF-BZ focusing on the regions marked by the red squares in $\mathbf{a}$ and $\mathbf{b}$ at a higher resolution. The images inserted in the upper right corners are their corresponding FFT patterns, the structure projections in HR-TEM images are along the $b$-axis and the structural models are inserted in the middle

observed crystal structures of the four COFs match well with the theoretical structure models along the $b$-axis. The images inserted in the upper right corners are the corresponding diffraction patterns of the four COFs obtained through fast Fourier transformation (FFT), further indicating single-crystal characteristics of these 2D COF flakes. The TEM investigation again corroborates that the as-prepared COFs hold the predicted triplepore and tetrad-pore framework structures.

Surface area and porosity measurements of the COFs. Porosities of the COFs were assessed by nitrogen adsorption-desorption measurements (Fig. 9). Their BET surface areas, derived from their nitrogen adsorption isotherms at $77 \mathrm{~K}$, are $673.8,1180.8$, 568.8 , and $864.3 \mathrm{~m}^{2} \mathrm{~g}^{-1}$ for Tri-COF-DAB, Tri-COF-BZ, TetraCOF-DAB, and Tetra-COF-BZ, respectively (Supplementary Fig. 16), and their corresponding total pore volumes (at $\mathrm{P} / \mathrm{P}_{\mathrm{o}}=$ 0.99 ) were estimated to be $1.02,1.15,0.34$, and $1.07 \mathrm{~cm}^{3} \mathrm{~g}^{-1}$. Derived from their theoretical $\mathrm{N}_{2}$ adsorption isotherms (Supplementary Fig. 17), the theoretical BET surface areas were calculated to be $1659.8,2388.4,1900.7$, and $2312.8 \mathrm{~m}^{2} \mathrm{~g}^{-1}$ for TriCOF-DAB, Tri-COF-BZ, Tetra-COF-DAB, and Tetra-COF-BZ, respectively (Supplementary Fig. 18). The low experimental values are likely attributed to framework flexibility of the COFs resulting from their large unit cell dimensions ${ }^{61}$. It should be noted that no informative information could be obtained from pore size distribution (PSD) analysis because no suitable model could be applied to these highly complexed COFs. Taking TetraCOF-BZ as an example, PSD analysis was carried out by trying all the kernels/pore models available from the instrument. However, not an appropriate model was found to be suitable for the COF, as the PSD profiles obtained did not have a definite meaning to the COFs (Supplementary Fig. 19), which may be due to the defects of the COFs or other effects. This result suggests the limitation of PSD on such complex framework materials. However, although PSD data are not available, the results of PXRD and HR-TEM provide compelling evidence for the formation of the pre-designed triple-pore and tetrad-pore COFs.

\section{Discussion}

In summary, a facile and general approach has been developed to construct 2D COFs with highly complicated topological structures. Implementation of this approach led to the successful construction of 2D COFs for which three or four different kinds of pores were integrated into one framework, increasing the structural complexity in the tessellations of $2 \mathrm{D}$ polymers to a much higher level. This work also demonstrates that the mathematically intricate tessellation patterns could be successfully challenged by synthetic chemistry. Since tessellating 2D COFs with different geometric shapes provides access to independent structural modification and functionalization of different parts of frameworks with high precision, it opens a promising way to fabricate advanced materials in which different functions can be highly integrated. With the development of more elaborate tessellation strategies, emergence of $2 \mathrm{D}$ polymers with much more structural complexity and multiple-functionalities can be expected in the near future. 

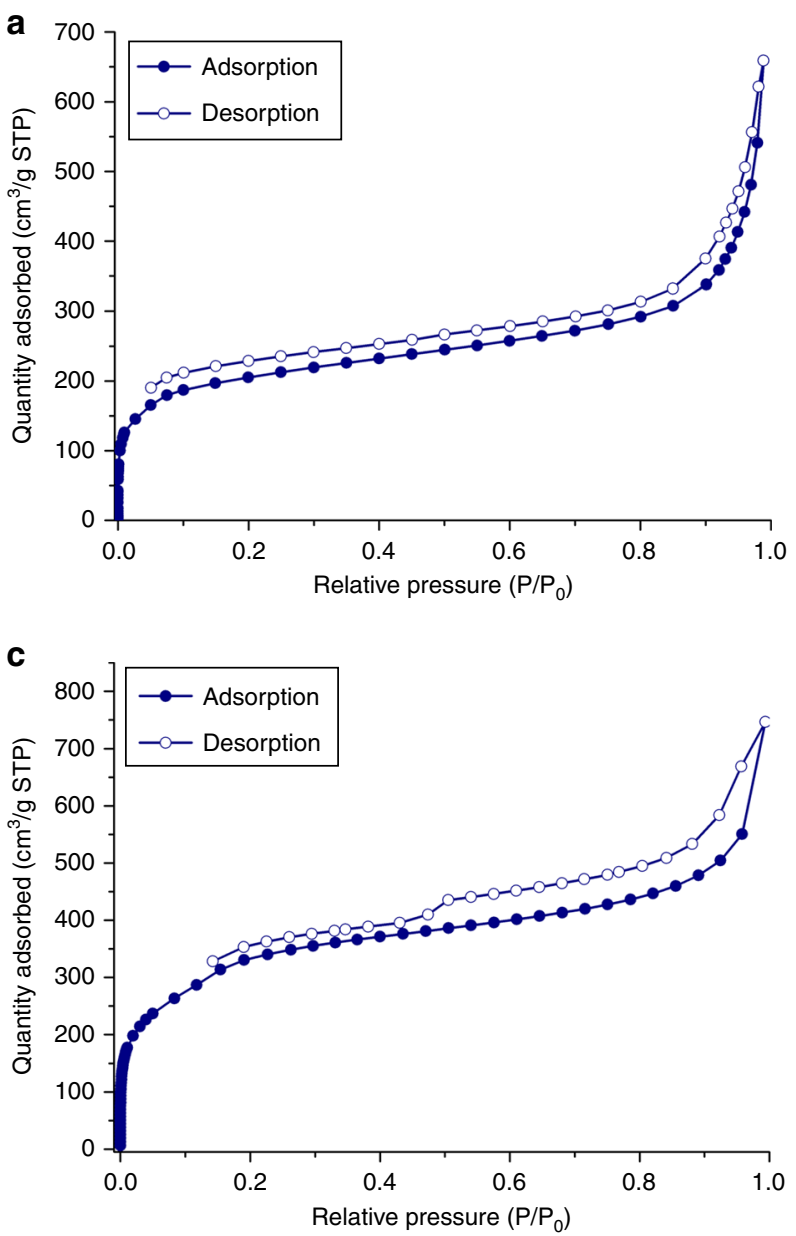
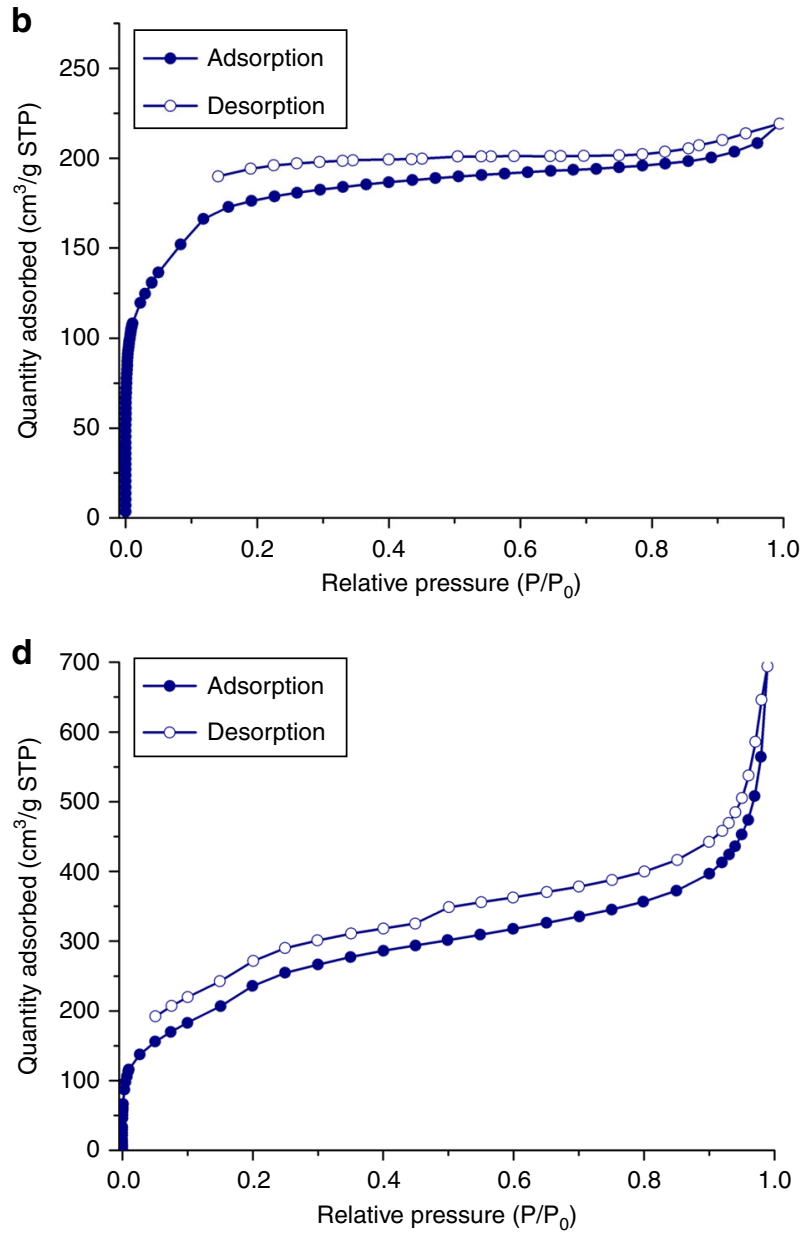

Fig. 9 Porosity measurements. $\mathrm{N}_{2}$ adsorption-desorption isotherms (77 K) of a Tri-COF-DAB, b Tetra-COF-DAB, c Tri-COF-BZ, and d Tetra-COF-BZ

\section{Methods}

Synthesis of Tri-COF-DAB. TPM ( $30.0 \mathrm{mg}, 0.043 \mathrm{mmol})$ and 1,4-diaminobenzene (DAB, $14.1 \mathrm{mg}, 0.130 \mathrm{mmol})$ were added into a mixture of 1,4-dioxane $(0.75 \mathrm{~mL})$, mesitylene $(0.75 \mathrm{~mL})$, and acetic acid (aq. $6 \mathrm{M}, 0.15 \mathrm{~mL})$ in a glass ampoule. The ampoule was sealed under vacuum after three freeze-pump-thaw cycles. Then the mixture was heated at $120^{\circ} \mathrm{C}$ without disturbance for $72 \mathrm{~h}$ to afford a yellow solid at the bottom of the tube. After being cooled to room temperature, the solvent was decanted and the solid was washed with anhydrous 1,4-dioxane and DCM for three times, respectively, and then dried under dynamic vacuum at $120^{\circ} \mathrm{C}$ for $4 \mathrm{~h}$ to afford a yellow powder $(31.7 \mathrm{mg}, 81 \%)$, which was insoluble in common organic solvents such as acetone, ethanol, and N, N-dimethylformamide. Anal. Calcd. For $\mathrm{C}_{64} \mathrm{H}_{39} \mathrm{~N}_{7}: \mathrm{C}, 84.84 ; \mathrm{H}, 4.34 ; \mathrm{N}, 10.82$. Found: $\mathrm{C}, 79.23 ; \mathrm{H}, 4.75 ; \mathrm{N}, 9.57$.

Synthesis of Tri-COF-BZ. TPM ( $25.0 \mathrm{mg}, 0.036 \mathrm{mmol})$ and benzidine (BZ, 20.0 $\mathrm{mg}, 0.108 \mathrm{mmol})$ were added into a mixture of 1,4 -dioxane $(1 \mathrm{~mL})$, mesitylene (1 $\mathrm{mL}$ ) and acetic acid (aq. $6 \mathrm{M}, 0.2 \mathrm{~mL}$ ) in a glass ampoule. The ampoule was sealed under vacuum after three freeze-pump-thaw cycles. Then the mixture was heated at $120^{\circ} \mathrm{C}$ without disturbance for $72 \mathrm{~h}$ to afford a yellow solid at the bottom of the tube. After being cooled to room temperature, the solvent was decanted and the solid was washed with anhydrous 1,4-dioxane and DCM for three times, respectively, and then dried under dynamic vacuum at $120^{\circ} \mathrm{C}$ for $4 \mathrm{~h}$ to afford a yellow powder $(30.0 \mathrm{mg}, 73 \%)$, which was insoluble in common organic solvents such as acetone, ethanol, and N, N-dimethylformamide. Anal. Calcd. For $\mathrm{C}_{82} \mathrm{H}_{51} \mathrm{~N}_{7}$ : C, 86.82; H, 4.53; N, 8.64. Found: C, 81.26; H, 4.98; N, 7.50.

Synthesis of Tetra-COF-DAB. FPM $(26.0 \mathrm{mg}, 0.031 \mathrm{mmol})$ and DAB $(10.0 \mathrm{mg}$, $0.092 \mathrm{mmol})$ were added into a mixture of 1,4-dioxane $(0.25 \mathrm{~mL})$, mesitylene $(0.75$ $\mathrm{mL}$ ) and acetic acid (aq. $6 \mathrm{M}, 0.1 \mathrm{~mL}$ ) in a glass ampoule. The ampoule was sealed under vacuum after three freeze-pump-thaw cycles. Then the mixture was heated at $120^{\circ} \mathrm{C}$ without disturbance for $72 \mathrm{~h}$ to afford a yellow solid at the bottom of the tube. After being cooled to room temperature, the solvent was decanted and the solid was washed with anhydrous 1,4-dioxane and DCM for three times, respectively, and then dried under dynamic vacuum at $120^{\circ} \mathrm{C}$ for $4 \mathrm{~h}$ to afford a yellow powder $(19.7 \mathrm{mg}, 60 \%)$, which was insoluble in common organic solvents such as acetone, ethanol, and N, N-dimethylformamide. Anal. Calcd. For $\mathrm{C}_{76} \mathrm{H}_{47} \mathrm{~N}_{7}$ : C, 86.26; H, 4.48; N, 9.27. Found: C, 80.05; H, 5.13; N, 7.02.

Synthesis of Tetra-COF-BZ. FPM (27.4 mg, $0.032 \mathrm{mmol})$ and BZ $(18.0 \mathrm{mg}, 0.098$ mmol) were added into a mixture of 1,4-dioxane $(1 \mathrm{~mL})$, mesitylene $(1 \mathrm{~mL})$ and acetic acid (aq. $9 \mathrm{M}, 0.2 \mathrm{~mL}$ ) in a glass ampoule. The ampoule was sealed under vacuum after three freeze-pump-thaw cycles. Then the mixture was heated at $120^{\circ}$ $\mathrm{C}$ without disturbance for $72 \mathrm{~h}$ to afford a yellow solid at the bottom of the tube After being cooled to room temperature, the solvent was decanted and the solid was washed with anhydrous 1,4-dioxane and DCM for three times, respectively, and then dried under dynamic vacuum at $120^{\circ} \mathrm{C}$ for $4 \mathrm{~h}$ to afford a yellow powder (37.0 $\mathrm{mg}, 90 \%$ ), which was insoluble in common organic solvents such as acetone, ethanol, and N, N-dimethylformamide. Anal. Calcd. For $\mathrm{C}_{94} \mathrm{H}_{59} \mathrm{~N}_{7}: \mathrm{C}, 87.76 ; \mathrm{H}$, 4.62; N, 7.62. Found: C, 84.79; H, 5.14; N, 7.25.

\section{Data availability}

All data supporting the findings of this study are available within the article, as well as the Supplementary Information file, or available from the corresponding authors on reasonable request.

Received: 1 June 2018; Accepted: 18 September 2019; Published online: 10 October 2019

\section{References}

1. Bechtle, S., Ang, S. F. \& Schneider, G. A. On the mechanical properties of hierarchically structured biological materials. Biomaterials 31, 6378-6385 (2010).

2. Fratzl, P. \& Weinkamer, R. Nature's hierarchical materials. Progr. Mater. Sci. 52, 1263-1334 (2007). 
3. Yang, X.-Y. et al. Hierarchically porous materials: synthesis strategies and structure design. Chem. Soc. Rev. 46, 481-558 (2017)

4. Lopez-Orozco, S., Inayat, A., Schwab, A., Selvam, T. \& Schwieger, W. Zeolitic materials with hierarchical porous structures. Adv. Mater. 23, 2602-2615 (2011).

5. Petkovich, N. D. \& Stein, A. Controlling macro- and mesostructures with hierarchical porosity through combined hard and soft templating. Chem. Soc. Rev. 42, 3721-3739 (2013).

6. Hartmann, M. Hierarchical Zeolites: a proven strategy to combine shape selectivity with efficient mass transport. Angew. Chem. Int. Ed. 43, 5880-5882 (2004).

7. Seo, M., Kim, S., Oh, J., Kim, S.-J. \& Hillmyer, M. A. Hierarchically porous polymers from hyper-cross-linked block polymer precursors. J. Am. Chem. Soc. 137, 600-603 (2015).

8. Parlett, C. M. A., Wilson, K. \& Lee, A. F. Hierarchical porous materials: catalytic applications. Chem. Soc. Rev. 42, 3876-3893 (2013).

9. Furukawa, H., Müller, U. \& Yaghi, O. M. "Heterogeneity within order" in metal-organic frameworks. Angew. Chem. Int. Ed. 54, 3417-3430 (2015).

10. Zhang, G., Presly, O., White, F., Oppel, I. M. \& Mastalerz, M. A shapepersistent quadruply interlocked giant cage catenane with two distinct pores in the solid state. Angew. Chem. Int. Ed. 53, 5126-5130 (2014).

11. Horne, C. E. Geometric symmetry in patterns and tilings. (Woodhead Publishing, 2000)

12. Novoselov, K. S. et al. Electric field effect in atomically thin carbon films Science 306, 666-669 (2004)

13. Geim, A. K. Graphene: status and prospects. Science 324, 1530-1534 (2009).

14. Novoselov, K. S. et al. Two-dimensional atomic crystals. Proc. Natl Acad. Sci. USA 102, 10451-10453 (2005).

15. Liu, H. et al. Phosphorene: an unexplored 2D semiconductor with a high hole mobility. ACS Nano 8, 4033-4041 (2014).

16. Côté, A. P. et al. Porous, crystalline, covalent organic frameworks. Science 310, 1166-1170 (2005).

17. Waller, P. J., Gándara, F. \& Yaghi, O. M. Chemistry of covalent organic frameworks. Acc. Chem. Res. 48, 3053-3063 (2015).

18. Colson, J. W. \& Dichtel, W. R. Rationally synthesized two-dimensional polymers. Nat. Chem. 5, 453-465 (2013).

19. Sakamoto, J., van Heijst, J., Lukin, O. \& Schlüter, A. D. Two-dimensional polymers: Just a dream of synthetic chemists? Angew. Chem. Int. Ed. 48, 1030-1069 (2009).

20. Doonan, C. J., Tranchemontagne, D. J., Glover, T. G., Hunt, J. R. \& Yaghi, O. M. Exceptional ammonia uptake by a covalent organic framework. Nat. Chem. 2, 235-238 (2010).

21. Zeng, Y., Zou, R. \& Zhao, Y. Covalent organic frameworks for $\mathrm{CO}_{2}$ capture. Adv. Mater. 28, 2855-2873 (2016)

22. Kuhn, P., Antonietti, M. \& Thomas, A. Porous, covalent triazine-based frameworks prepared by ionothermal synthesis. Angew. Chem. Int. Ed. 47, 3450-3453 (2008).

23. Huang, N., Wang, P., Addicoat, M. A., Heine, T. \& Jiang, D. Ionic covalent organic frameworks: design of a charged interface aligned on $1 \mathrm{D}$ channel walls and its unusual electrostatic functions. Angew. Chem. Int. Ed. 56, 4982-4986 (2017).

24. Oh, H. et al. A cryogenically flexible covalent organic framework for efficient hydrogen isotope separation by quantum sieving. Angew. Chem. Int. Ed. 52, 13219-13222 (2013).

25. Kang, Z. et al. Mixed matrix membranes (MMMs) comprising exfoliated 2D covalent organic frameworks (COFs) for efficient $\mathrm{CO}_{2}$ separation. Chem. Mater. 28, 1277-1285 (2016)

26. Shinde, D. B., Kandambeth, S., Pachfule, P., Kumar, R. R. \& Banerjee, R. Bifunctional covalent organic frameworks with two dimensional organocatalytic micropores. Chem. Commun. 51, 310-313 (2015).

27. Xu, H.-S., Ding, S.-Y., An, W.-K., Wu, H. \& Wang, W. Constructing crystalline covalent organic frameworks from chiral building blocks. J. Am. Chem. Soc. 138, 11489-11492 (2016).

28. Zhang, J., Han, X., Wu, X., Liu, Y. \& Cui, Y. Multivariate chiral covalent organic frameworks with controlled crystallinity and stability for asymmetric catalysis. J. Am. Chem. Soc. 139, 8277-8285 (2017).

29. Sun, Q., Aguila, B. \& Ma, S. A bifunctional covalent organic framework as an efficient platform for cascade catalysis. Mater. Chem. Front. 1, 1310-1316 (2017).

30. Pachfule, P. et al. Diacetylene functionalized covalent organic framework (COF) for photocatalytic hydrogen generation. J. Am. Chem. Soc. 140, 1423-1427 (2018).

31. Bai, L. et al. Nanoscale covalent organic frameworks as smart carriers for drug delivery. Chem. Commun. 52, 4128-4131 (2016).

32. Vyas, V. S. et al. Exploiting noncovalent interactions in an imine-based covalent organic framework for quercetin delivery. Adv. Mater. 28, 8749-8754 (2016).
33. Ding, S.-Y. et al. Thioether-based fluorescent covalent organic framework for selective detection and facile removal of mercury(II). J. Am. Chem. Soc. 138 , 3031-3037 (2016)

34. Peng, Y. et al. Ultrathin two-dimensional covalent organic framework nanosheets: preparation and application in highly sensitive and selective DNA detection. J. Am. Chem. Soc. 139, 8698-8704 (2017).

35. Das, G. et al. Chemical sensing in two dimensional porous covalent organic nanosheets. Chem. Sci. 6, 3931-3939 (2015).

36. Chandra, S. et al. Phosphoric acid loaded azo $(-\mathrm{N}=\mathrm{N}-)$ based covalent organic framework for proton conduction. J. Am. Chem. Soc. 136, 6570-6573 (2014).

37. $\mathrm{Xu}, \mathrm{H} ., \mathrm{Tao}, \mathrm{S}$. \& Jiang, D. Proton conduction in crystalline and porous covalent organic frameworks. Nat. Mater. 15, 722-726 (2016).

38. Dogru, M. \& Bein, T. On the road towards electroactive covalent organic frameworks. Chem. Commun. 50, 5531-5546 (2014).

39. Chen, L. et al. Photoelectric covalent organic frameworks: converting open lattices into ordered donor-acceptor heterojunctions. J. Am. Chem. Soc. 136, 9806-9809 (2014)

40. Huang, N., Ding, X., Kim, J., Ihee, H. \& Jiang, D. A photoresponsive smart covalent organic framework. Angew. Chem. Int. Ed. 54, 8704-8707 (2015).

41. DeBlase, C. R., Silberstein, K. E., Truong, T.-T., Abruña, H. D. \& Dichtel, W. R. $\beta$-Ketoenamine-linked covalent organic frameworks capable of pseudocapacitive energy storage. J. Am. Chem. Soc. 135, 16821-16824 (2013).

42. Liao, H. et al. A 2D porous porphyrin-based covalent organic framework for sulfur storage in lithium-sulfur batteries. J. Mater. Chem. A 4, 7416-7421 (2016).

43. Wang, S. et al. Exfoliation of covalent organic frameworks into few-layer redox-active nanosheets as cathode materials for lithium-ion batteries. J. Am. Chem. Soc. 139, 4258-4261 (2017).

44. $\mathrm{Du}, \mathrm{Y}$. et al. Ionic covalent organic frameworks with spiroborate linkage. Angew. Chem. Int. Ed. 55, 1737-1741 (2016).

45. Segura, J. L., Mancheño, M. J. \& Zamora, F. Covalent organic frameworks based on Schiff-base chemistry: synthesis, properties and potential applications. Chem. Soc. Rev. 45, 5635-5671 (2016)

46. Ding, S.-Y. \& Wang, W. Covalent organic frameworks (COFs): from design to applications. Chem. Soc. Rev. 42, 548-568 (2013).

47. Beuerle, F. \& Gole, B. Covalent organic frameworks and cage compounds: design and applications of polymeric and discrete organic scaffolds. Angew. Chem. Int. Ed. 57, 4850-4878 (2018).

48. Zhou, T.-Y., Xu, S.-Q., Wen, Q., Pang, Z.-F. \& Zhao, X. One-step construction of two different kinds of pores in a 2D covalent organic framework. J. Am. Chem. Soc. 136, 15885-15888 (2014).

49. Pang, Z.-F. et al. Construction of covalent organic frameworks bearing three different kinds of pores through the heterostructural mixed linker strategy. J. Am. Chem. Soc. 138, 4710-4713 (2016)

50. Xu, S.-Q., Zhan, T.-G., Wen, Q., Pang, Z.-F. \& Zhao, X. Diversity of covalent organic frameworks (COFs): a $2 \mathrm{D}$ COF containing two kinds of triangular micropores of different sizes. ACS Macro Lett. 5, 99-102 (2016).

51. Tian, Y. et al. Two-dimensional dual-pore covalent organic frameworks obtained from the combination of two $\mathrm{D}_{2 \mathrm{~h}}$ symmetrical building blocks. Chem. Commun. 52, 11704-11707 (2016)

52. Yin, Z.-J. et al. Ultrahigh volatile iodine uptake by hollow microspheres formed from a heteropore covalent organic framework. Chem. Commun. $\mathbf{5 3}$ 7266-7269 (2017)

53. Jin, Y., Hu, Y. \& Zhang, W. Tessellated multiporous two-dimensional covalent organic frameworks. Nat. Rev. Chem. 1, 0056 (2017).

54. Qian, C. et al. Toward covalent organic frameworks bearing three different kinds of pores: the strategy for construction and COF-to-COF transformation via heterogeneous linker exchange. J. Am. Chem. Soc. 139, 6736-6743 (2017).

55. Liang, R.-R., Xu, S.-Q., Pang, Z.-F., Qi, Q.-Y. \& Zhao, X. Self-sorted poreformation in the construction of heteropore covalent organic frameworks based on orthogonal reactions. Chem. Commun. 54, 880-883 (2018)

56. Yang, H. et al. Mesoporous 2D covalent organic frameworks based on shapepersistent arylene-ethynylene macrocycles. Chem. Sci. 6, 4049-4053 (2015).

57. Baldwin, L. A., Crowe, J. W., Shannon, M. D., Jaroniec, C. P. \& McGrier, P. L. 2D covalent organic frameworks with alternating triangular and hexagonal pores. Chem. Mater. 27, 6169-6172 (2015).

58. Sun, Q., Aguila, B., Lan, P. C. \& Ma, S.Tuning pore heterogeneity in covalent organic frameworks for enhanced enzyme accessibility and resistance against denaturants. Adv. Mater. 31, 1900008 (2019).

59. Qian, C., Xu, S.-Q., Jiang, G.-F., Zhan, T.-G. \& Zhao, X. Precision construction of $2 \mathrm{D}$ heteropore covalent organic frameworks by a multiple-linking-site strategy. Chem. Eur. J. 22, 17784-17789 (2016).

60. Zhu, Y., Wan, S., Jin, Y. \& Zhang, W. Desymmetrized vertex design for the synthesis of covalent organic frameworks with periodically heterogeneous pore structures. J. Am. Chem. Soc. 137, 13772-13775 (2015).

61. Zhao, C. et al. Urea-linked covalent organic frameworks. J. Am. Chem. Soc. 140, 16438-16441 (2018) 


\section{Acknowledgements}

We thank the National Science Fund for Distinguished Young Scholars of China (No. 21725404) and the Strategic Priority Research Program of the Chinese Academy of Sciences (Grant No. XDB20000000) for financial support.

\section{Author contributions}

X.Z., R.L., and S.X. conceived the project. X.Z. supervised the project. R.L. and S.X. designed and performed the experiments for the synthesis and characterizations of the monomers and COFs, including NMR, IR, TGA, SEM, PXRD, gas adsorption and structure simulations. L.Z., P.C., and J.S. performed the TEM experiments, part PXRD experiments and analyzed the data. R.A aided in the synthesis of the COFs, Q.Q. aided in the structure simulations, and F.C. aided in the synthesis of some monomers. All the authors discussed the results and wrote the manuscript.

\section{Competing interests}

The authors declare no competing interests.

\section{Additional information}

Supplementary information is available for this paper at https://doi.org/10.1038/s41467019-12596-6.

Correspondence and requests for materials should be addressed to J.S. or X.Z.
Peer review information Nature Communications thanks Wei Zhang and the other, anonymous, reviewer(s) for their contribution to the peer review of this work.

Reprints and permission information is available at http://www.nature.com/reprints

Publisher's note Springer Nature remains neutral with regard to jurisdictional claims in published maps and institutional affiliations.

(c) (i) Open Access This article is licensed under a Creative Commons Attribution 4.0 International License, which permits use, sharing, adaptation, distribution and reproduction in any medium or format, as long as you give appropriate credit to the original author(s) and the source, provide a link to the Creative Commons license, and indicate if changes were made. The images or other third party material in this article are included in the article's Creative Commons license, unless indicated otherwise in a credit line to the material. If material is not included in the article's Creative Commons license and your intended use is not permitted by statutory regulation or exceeds the permitted use, you will need to obtain permission directly from the copyright holder. To view a copy of this license, visit http://creativecommons.org/ licenses/by/4.0/.

(C) The Author(s) 2019 